R. J. Cohen and W. T. Sullivan, III, eds.

\title{
Techniques for Coping with Radio Frequency Interference
}

\author{
J. R. Fisher \\ NRAO, P.O. Box 2, Green Bank, WV, USA
}

\begin{abstract}
As a complement to spectrum management efforts by radio astronomers a number of observatories and research groups around the world have begun looking into technical solutions to the problem of separating weak cosmic radiation from man-made radio signals. Some of the technical research now getting underway includes: high dynamic range receivers, low-noise superconducting filters, passive digital filtering, adaptive filters, adaptive sidelobe nulling, multi-feed correlation of RFI, and various techniques for signal blanking.

Increased technical support to spectrum management can also be provided in the form of accurate and statistically significant characterization of the radio environment, empirical and theoretical improvement of over-the-horizon propagation models, and timely measurements of spurious radiation falling in the protected radio astronomy bands.

Finally, credibility of our spectrum management effort can only be maintained by making sure that local radiation under the control of our radio observatories is in compliance with the field strength limits of Recommendation ITU-R R.A.769. This requires sensitive radiation measurements and often shielding of digital equipment, microwave ovens, test equipment, local oscillators, etc.
\end{abstract}

\section{Introduction}

The title of this paper was chosen to emphasize radio frequency interference (RFI) excision and cancellation techniques, but let me expand the topic a bit to include technical support for spectrum management and the measures that we can take to maintain a clean environment in the vicinity of our radio observatories. To sustain a credible presence in the national and international spectrum management agencies the radio astronomy community must show that it is allocating significant resources to quantitatively monitor the radio spectrum and that it is keeping up with the state of the art in RFI reduction. Our current effort in these areas is too small.

Radio astronomy and passive remote sensing are unique amongst the radio services in several ways. They are mostly receive-only; their received signals are typically from three to six orders of magnitude weaker that those of the communications services; and their signals from natural sources are spread throughout the entire electromagnetic spectrum. These last two aspects of our science make the task of monitoring the radio spectrum a daunting but not impossible one. 
The allocation of resources for RFI control can be divided into two categories: organization of current technologies and research and development. The first includes an adequate number of monitoring stations to maintain cleanliness of the exclusive radio astronomy bands, to verify spectrum sharing agreements, to enforce quiet zone and coordination zone agreements, and to evaluate new sites for radio observatories. It also includes thorough shielding and filtering of RFI sources in the vicinities of radio telescopes and the engineering of receivers that can tolerate signals from other radio services.

Research and development of RFI excision techniques are also essential. The protected radio astronomy bands cannot possibly cover all frequencies of importance to the science. Radio astronomers have been resourceful in finding remote sites and observing at times and frequencies that are not fully occupied by the allocated radio services, but the increasing density of spectrum use is making this more problematic. We need to more efficiently separate signals in the spatial domain with sidelobe cancelling and null steering techniques that have already been developed by radar and acoustic engineers. The extremely low signal-to-noise requirements of radio astronomy demand, however, a significant extension of the current state of the art.

\section{Support for Spectrum Management}

I think that it is fair to say that radio astronomy's spectrum managers have been inadequately supported by field measurements of the radio environment around our observatories. Reports of interference to observations in the protected bands tend to be anecdotal, qualitative, and very sporadic. Although we can fully justify the harmful interference levels set out in Recommendation ITU-R R.A.769, the case is seen as a largely hypothetical one by many of our competitors for spectrum space. If radiation in the protected bands were reported in a more timely and systematic manner, our spectrum managers could make a stronger argument for better protection to the rule-making agencies.

The enormous increase in satellite services for navigation and communication has created a strong demand for downlink spectrum allocations near protected radio astronomy bands. Because satellites are line-of-sight to radio telescopes, their signals can be quite strong. This puts stringent requirements on the satellite transmitters to avoid producing harmful spurious radiation in the radio astronomy bands. To verify that the satellite transmitters comply with these requirements, radio astronomers have had to measure the spurious radiation during the satellite's test or commissioning phase. In the case of the Iridium satellite system this took many man-months of engineering effort to refit receivers, and the tests occupied a number of days of radio telescope time that would otherwise have been used for astronomy. We can expect a demand for more of these measurements in the future. It will behove us to invest in several dishes in the 10-15 meter class with receivers and signal processing back-ends to avoid using valuable time on our best radio telescopes. Furthermore, if such measurements were made routine, they would serve as a monitor of compliance throughout the lifetime of many satellites.

In recent years the National Radio Quiet Zone around NRAO's Green Bank observatory has been challenged several times on the basis of dubious propa- 
gation calculations used to request limits on the radiation from transmitters installed in the Quiet Zone. Different propagation models give quite different answers in some instances. The only way to conclusively resolve each challenge is with statistically significant measurements over a range of foliation and atmospheric conditions. We are just now designing measurement facilities for this purpose, and it will be several years before we have a good understanding of the accuracy of our propagation model.

\section{Sensitivity}

One occasionally hears the lament that an RFI monitoring station cannot hope to reach the sensitivity of a radio telescope because of the latter's very low noise receivers and integrating radiometers. That is true if all one has is a simple antenna and a common spectrum analyzer, but we need to do much better.

The harmful limits set by Recommendation ITU-R R.A.769 assume an isotropic gain for the far sidelobes of a radio telescope with a typical system temperature of $20 \mathrm{~K}$. A monitor antenna and uncooled preamp necessarily has a noise temperature of about $300 \mathrm{~K}$, but this can be compensated by a reasonably modest $12 \mathrm{~dB}$ of antenna gain in the direction of the RFI source.

There is no reason that we cannot use the signal processing power of radio astronomy spectrometers and continuum radiometers on the monitor antenna signal to realize the same integration times and spectral and time resolutions as are used for astronomical measurements. Ideally, one would like to build a small, dedicated integrating spectrometer for RFI detection and monitoring, but various time or channel sharing schemes can also be made to work with existing equipment. Digital spectrometer bandwidths of several tens of $\mathrm{MHz}$ are now relatively inexpensive.

Our monitor station plans for the new 100-m Green Bank Telescope (GBT) include a set of antennas near the top of the feed support arm which will share the alternate LO synthesizer and one or more of the eight fiber optic IF channels to the control room. An old $2 \times 40 \mathrm{MHz}$ bandwidth FFT spectrometer will be available most of the time for RFI measurements while the telescope is using a new $800 \mathrm{MHz}$ correlator. A second monitor station near the lab will have a similar set of antennas and three optical fiber links to the GBT control room and the FFT spectrometer.

\section{Control of Our Own Radiation}

A radio observatory requires thousands of pieces of electronic and computing equipment, each with real potential for interference to astronomical measurements. Many of us have experienced this painful fact. As a practical matter and to demonstrate that we are serious about the radiation limits that we ask of other radio services, we are obligated to suppress the radiation from our own equipment to the levels set by Recommendation ITU-R R.A.769, at least in the radio astronomy bands but, ideally, at all of our observing frequencies.

Retrofitting an observatory for RFI suppression is an expensive and timeconsuming business. The DRAO observatory at Penticton is a notable success story. They have gone to considerable lengths to shield computers, fax machines, 
correlators, and even a postage scale to clean up their environment around $21 \mathrm{~cm}$ wavelength, where they are making a large scale survey of the sky. Similarly, the VLA went to considerable expense and effort to suppress radiation from equipment in their antennas to meet the requirements of new 330 and $74 \mathrm{MHz}$ receivers. The lesson to be learned is that RFI control must be an integral part of an observatory design from the very beginning.

At Green Bank we have the fortunate opportunity to partially start from scratch with the new GBT and its control room. An important part of this new construction has been a program to measure most of the new electronic equipment that will be installed on or near the GBT. These measurements are done in a shielded anechoic chamber. The isolated environment and occasional use of an FFT spectrometer and specially programmed continuum signal processing have allowed us to measure RFI emissions that are commensurate with the limits of Recommendation ITU-R R.A.769. The field strength measurements for each piece of equipment are then converted to a power density at the GBT feed, using free space loss and any shielding external to the unit. Where necessary the equipment is sent back to the lab for further shielding and filtering. Very few pieces have escaped the need for additional suppression, and even apparently innocuous items like linear power supplies have been found to be a source of substantial RFI.

Other measures that have been implemented in connection with GBT construction are a $60 \mathrm{~dB}$-shielded secondary receiver room, a $60 \mathrm{~dB}$-shielded equipment and control room removed about two kilometers from the telescope, and transfer of computers and other equipment for the other telescopes to the shielded control room. The biggest problems that remain are many pieces of computing and test equipment in an unshielded lab on site. These will be slowly addressed on a room by room and unit by unit basis.

\section{Site Characterization}

To my knowledge there is very little quantitative information that allows us to compare the RFI environments of the many radio observatories around the world. Hence, we have essentially no basis from which to begin a site evaluation for future radio telescopes, most notably the Square Kilometer Array (SKA).

Part of the problem is that we have never established a standard measurement procedure, primarily because making accurate and statistically representative field strength measurements over several decades of frequency is a large task. An informal group that met at the SKA Workshop in Dwingeloo, the Netherlands, in April 1999 is making a modest beginning at establishing measurement guidelines or standards. A number of observatories have most of the facilities needed to begin a comparative measurement program, but it remains to be seen how much support this effort will gather. It is important to the radio astronomical community that substantial resources should now be devoted to this task as an investment in future RFI control and spectrum management. 


\section{RFI Excision}

We commonly think of RFI in the frequency domain, but from an observer's viewpoint the location of interference is better described in at least four dimensions: frequency, time, and two directional coordinates. If a source of cosmic radiation does not coincide in all four coordinates with any RFI sources, we can, in principle, isolate the cosmic radiation for measurement. Of course, in practice this is a challenging and imperfect task.

In this four-coordinate phase space the electromagnetic spectrum is very sparsely populated. At a given observing location one frequency may be occupied by only one RFI source in a specific direction, or the RFI source may cover a continuum of frequencies but have a small duty cycle. In many cases the cosmic radiation may be isolated by placing a single null on the interfering source in only one of the four coordinates. All forms of RFI excision do just that in one way or another.

For the following brief discussion of excision techniques we assume that the frequency of the cosmic radiation is occupied by an interfering signal so that we must separate the two in one of the time and spatial coordinates.

\subsection{Blanking (Temporal Excision)}

The simplest method of RFI excision is to divide the observational data into a modest number of time intervals and throw away, based on visual inspection, data that is corrupted by interference. When the number of time bins gets to be more than a few hundred, this process must be automated, but matching the ability of the human eye at picking out anomalies in data is a difficult task. The trick is to find a property of the interference that differs mathematically from random noise and from the intended astronomical signal.

If the interference has a sufficiently low duty cycle it can be detected in the data by comparing the instantaneous power to a running mean or median of surrounding time samples. This has been successfully demonstrated by Peter Fridman at the Special Astrophysical Observatory and NFRA on RATAN-600 data and by Morgan and Fisher (1977) on data from the late NRAO 91-m telescope. To be effective the data sampling interval must be short enough to resolve the interference temporal structure, and the mean or median interval must be long enough to get a good sample of the data in the absence of interference. Hence, the sampling and averaging time scales must be carefully matched to the temporal properties of the RFI, and one must be careful that the exciser does not affect the astronomical measurements. The necessity for manual optimization has been a deterrent to implementation of this type of RFI excision. A good user interface with sufficient display of the results of the excision process would probably help. A more robust excision algorithm which used as many as $10^{5}$ time samples in real-time detection of outliers in a $22 \mathrm{MHz}$ interferometer was reported by Kasper et al (1982).

A very different approach to detection and blanking has been simulated for a signal which is correlated in the outputs of a synthesis array such as the Westerbork radio telescope (Leshem and van der Veen 1999). When the correlated signal rises to a level that is above anything expected from an astronomical source, the data integration process is suspended until the correlated signal 
falls below threshold. This detection scheme has the considerable advantage of a known zero-interference reference level (zero correlated signal), but, like all blanking methods, a successful astronomical measurement depends on having a significant fraction of interference-free time.

\subsection{Spatial Nulling}

Since total integration time is part of the sensitivity equation we would prefer not to discard any data. Also, we need an excision technique that works when RFI is present all or most of the time at the frequencies of the cosmic signals. To accomplish this we must isolate and discard the interference in the spatial domain.

The ideal spatial isolation would be a radio telescope with no sidelobes, but this is a practical impossibility. The next best thing is to create nulls in the sidelobe response in the directions of interfering signals while being careful not to affect the gain of the antenna's main beam. In the signal processing literature (e.g., Widrow and Stearns 1985) the technique of spatial nulling is often divided into two categories, interference cancellation and null steering, but they really amount to the same thing. The first is usually found in connection with acoustics and single antennas, and the latter is found in the antenna array literature.

The basic idea is that a sample of the interfering signal is added to the $\mathrm{RF}$ path of a radio telescope, but in opposite phase and equal amplitude to the original RFI signal that entered through the telescope's sidelobes. In the case of a paraboloid antenna the interference sample can be obtained by an auxiliary antenna whose gain is somewhat higher than the sidelobe gain. In an antenna array the cancelling signal is generated with a slight modification of the complex weights of the array element signals that make up the mainbeam-forming network. An auxiliary antenna could also be used with an array. One advantage of an auxiliary antenna is that it can have very little gain in the direction of the astronomical source, and hence its signal will have little effect on the main beam gain. It is not clear at this point whether this is an important advantage in practice.

Because the radio telescope and possibly the interfering source are not stationary, the phase and amplitude of the cancelling signal must be made to track the changing amplitude and phase of the interference entering through the sidelobes. This requires a servo loop that maintains cancellation under all expected conditions, hence the names adaptive cancellation and adaptive null steering. Also, because most interfering signals are not monochromatic, and the delays and filter characteristics in the main and auxiliary signal paths are necessarily different, the auxiliary signal must have its phase and amplitude conditioned as a function of frequency. This requires an adaptive filter, usually in the form of a tapped delay network, in the auxiliary signal path.

Figure 1, which is adapted from a figure in Barnbaum and Bradley (1998), shows the block diagram of a simple adaptive cancelling arrangement. The servo criterion for the adaptive algorithm is to minimize the output of the summing network by adjusting the weights of the tapped delay network. More than one interfering signal can be cancelled with this simple arrangement as long as the signals are at different frequencies and there are a sufficient number of taps in the delay network. Lab measurements have demonstrated at least $70 \mathrm{~dB}$ of 


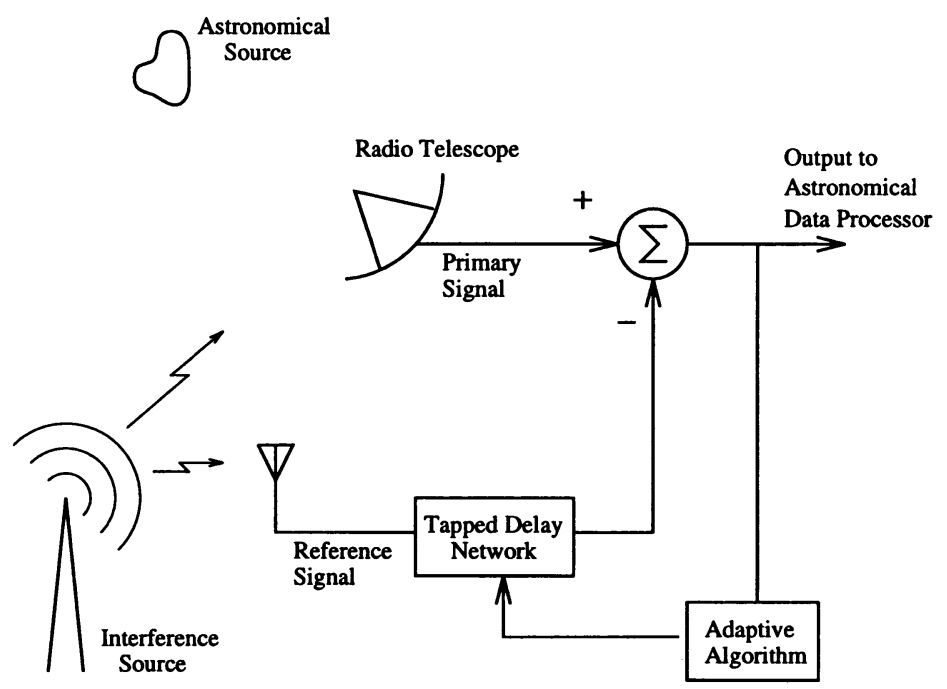

Figure 1. Basic adaptive cancellation scheme.

signal cancellation with this scheme, and preliminary tests on the NRAO 43-m telescope showed suppressions on FM broadcast signals of up to $20 \mathrm{~dB}$. A number of improvements in the adaptive hardware are required before attempting to understand the fundamental limits of this technique for astronomical observations. A second reference antenna and adaptive filter will be needed to cancel signals in the orthogonal polarization.

One question that remains to be answered is how effective the adaptive cancellation will be on weak RFI signals that are still strong enough to affect astronomical measurements. Barnbaum and Bradley showed that the signal-tonoise ratio of the interfering signal in the auxiliary channel must be $10 \mathrm{~dB}$ for every $20 \mathrm{~dB}$ of suppression required. This is due to the small DC offset in the error signal caused by white noise in the auxiliary channel. My intuition tells me that there is another limit imposed by the noise in the main channel in the sense that the adaptive servo cannot set the delay tap coefficients any more accurately than is allowed by the integration time of the adaptive loop. If the astronomical integration time is much longer than the inverse of the loop bandwidth, we may see a residual RFI signal that cannot be cancelled by the adaptive filter. We plan to test this with the current hardware.

The adaptive array null steering technique is being investigated by the Square Kilometer Array development group at the NFRA in Holland. They have prototyped an eight element array and demonstrated the basic concept in their antenna range (Goris 1997). This work has posed a number of interesting 
questions that need to be answered before we see a practical implementation of null steering in a radio astronomy array.

Another important question that needs to be answered for any RFI cancelling scheme is how much does the adaptive servo affect the stability of the astronomical measurement? We know that the auxiliary signal channel will add noise with frequency structure and that adaptive nulling in an array has the potential for affecting the main beam gain and shape. Can these be sufficiently controlled with constraints on the adaptive algorithm or by suitable calibration methods?

\subsection{Correlation Removal}

Ekers and Sault (1997) have suggested a method for subtracting interference from single dish data using the cross correlation of signals from elements in a focal plane array. If one assumes that the astronomical signals are essentially uncorrelated between the array elements and that the number $N$ of interfering sources and the number $M$ of array elements are related as $N \leq 2 M-1$, then the gain of each element in the direction of the interfering sources can be computed using a method analogous to self-calibration of synthesis array data. Using these computed gains, the interfering signals may be subtracted from the autocorrelation (total power) of each element. Initial trials of this technique with the Parkes 13-beam, 21-cm receiver were encouraging, but, as with so many interference subtraction attempts, one is left with a puzzle about why it works less well than expected.

Ekers and Sault pointed out that a more accurate interference subtraction might be obtained if some of the receiver array elements were deliberately pointed at the sources of RFI. This is somewhat analogous to using several reference antennas to sample the interference for the purpose of nulling with an adaptive filter as described above.

Interference subtraction with correlation data is a power subtraction. Hence, it requires an accurate gain calibration to be effective. This may be a disadvantage when compared to the adaptive filter technique, which does its subtraction in the voltage domain for which there is a definitive null criterion for success. Conversely, the correlation technique might lend itself to a global solution of interference subtraction, using all of the data in an astronomical integration to overcome the noise limitation of the adaptive filter loop bandwidth. At this point these are just speculations on my part.

\subsection{Other Experiments}

Jon Bell (ATNF), Rick Smegal (SETI Institute), and collaborators have recorded baseband data of interfering sources at the Australia Telescope Compact Array (ATCA) and are in the process of recording correlation data of interference on the Parkes 64-meter antenna. The Compact Array data are now available on CDROM for use by anyone who would like to experiment with RFI subtraction algorithms. The Parkes data will be similarly published.

One distinguishing feature of all man-made signals is that they are fully polarized at their point of origin. Single path propagation effects (reflection, diffraction, and refraction) and the response of a radio telescope's sidelobes will change this polarization to a different elliptical polarization, but the polarization 
fraction should remain $100 \%$. To test this I tried recording the four Stokes parameters of the many spectral features of an $80 \mathrm{MHz}$ commercial TV signal with the NRAO 43-m telescope. Subtraction of the root sum of the polarized Stokes parameters from the total intensity, $I-\sqrt{Q^{2}+U^{2}+V^{2}}$, should eliminate the TV signal. The measured results were that the TV signal was reduced between 0 and $20 \mathrm{~dB}$, depending on the spectral feature of the signal. This leads me to suspect that multipath propagation coupled with the wide TV signal bandwidth is causing depolarization of the signal. This could be tested by doing a similar experiment on narrow band signals at higher frequencies, for which depolarization should be less severe. If multipath propagation is a prevalent phenomenon, any adaptive cancelling of correlation subtraction techniques will need to take this into account.

My guess is that we must understand propagation effects on RFI signals before our cancelling and null steering techniques can reach their full potential. Some of what we need is in the engineering literature, but the unique nature of radio astronomy measurement will likely require advances in the general understanding of propagation. This may very well become an interesting cross-disciplinary research area.

\section{Communication of Research}

My apology to anyone in radio astronomy whose work in the areas of RFI monitoring and excision I have not mentioned. Part of this is due to my incomplete survey of the literature, but a bigger problem is that many efforts are in internal reports or possibly never written up. Much of this work deserves a wider distribution, even if not at the level of a journal article. To this end we are starting a Internet web site and email distribution list to which we invite contributions and subscription. You can contact me at rfisher@nrao.edu or Jon Bell at Jon.Bell@atnf.csiro.au for more information.

\section{References}

Barnbaum, C. and Bradley, R. F. 1998, AJ, 115, 2598

Ekers, R. and Sault, B. 1997, "Notes on estimation of interference using arrays", ATNF Internal Memo, 4 April 1997

Goris, M. J., 1997, "The adaptive beamformer of the SKAI adaptive antenna demonstrator", NFRA Technical Report - 459/MG/CB/V2.2

Kasper, B. L., Chute, F. S. and Routledge, D. 1982, MNRAS, 199, 345

Leshem, A. and van der Veen, A-J., 1999, "Detection and blanking of GSM interference in radio-astronomical observations", IEEE Workshop on Signal Processing Advances in Wireless Communication (SPAWC), Annapolis, Maryland, May 1999

Morgan J. V. and Fisher, J. R. 1977, "An impulse noise suppressor for continuum radiometry", NRAO Electronics Division Internal Report, No. 178

Widrow, B. and Stearns, S. D. 1985, Adaptive Signal Processing (Englewood Cliffs, NJ; Prentice Hall) 